\title{
BMJ Open Quantitative coronary angiography versus intravascular ultrasound guidance for drug-eluting stent implantation (GUIDE-DES): study protocol for a randomised controlled non-inferiority trial
}

\author{
Pil Hyung Lee, ${ }^{1}$ Soon Jun Hong (D) , ${ }^{2}$ Hyun-Sook Kim, ${ }^{3}$ Young won Yoon, ${ }^{4}$ \\ Jong-Young Lee, ${ }^{5}$ Seung-Jin Oh, ${ }^{6}$ Soo-Jin Kang, ${ }^{1}$ Young-Hak Kim, ${ }^{1}$ \\ Seong-Wook Park, ${ }^{1}$ Seung-Whan Lee, ${ }^{1}$ Cheol Whan Lee (1) , ${ }^{1}$ The GUIDE-DES Trial \\ Research Group
}

To cite: Lee PH, Hong SJ, Kim H-S, et al. Quantitative coronary angiography versus intravascular ultrasound guidance for drug-eluting stent implantation (GUIDEDES): study protocol for a randomised controlled noninferiority trial. BMJ Open 2022;12:e052215. doi:10.1136/ bmjopen-2021-052215

- Prepublication history and additional supplemental material for this paper are available online. To view these files, please visit the journal online (http://dx.doi.org/10.1136/ bmjopen-2021-052215)

Received 18 April 2021 Accepted 22 December 2021

Check for updates

(c) Author(s) (or their employer(s)) 2022. Re-use permitted under CC BY-NC. No commercial re-use. See rights and permissions. Published by BMJ.

For numbered affiliations see end of article.

Correspondence to

Dr Cheol Whan Lee;

cheolwlee@amc.seoul.kr

\section{ABSTRACT}

Introduction Angiography remains the gold standard for guiding percutaneous coronary intervention (PCl). However, it is prone to suboptimal stent results due to the visual estimation of coronary measurements. Although the benefit of intravascular ultrasound (IVUS)-guided PCl is becoming increasingly recognised, IVUS is not affordable for many catheterisation laboratories. Thus, a more practical and standardised angiography-based approach is necessary to support stent implantation.

Methods and analysis The Quantitative Coronary Angiography versus Intravascular Ultrasound Guidance for Drug-Eluting Stent Implantation trial is a randomised, investigator-initiated, multicentre, open-label, noninferiority trial comparing the quantitative coronary angiography (QCA)-guided PCI strategy with IVUS-guided $\mathrm{PCl}$ in all-comer patients with significant coronary artery disease. A novel, standardised, QCA-based PCI protocol for the QCA-guided group will be provided to all participating operators, while the $\mathrm{PCl}$ optimisation criteria will be predefined for both strategies. A total of 1528 patients will be randomised to either group at a 1:1 ratio. The primary endpoint is the 12-month cumulative incidence of target-lesion failure defined as a composite of cardiac death, target-vessel myocardial infarction or ischaemiadriven target-lesion revascularisation. Clinical follow-up assessments are scheduled at 1, 6 and 12 months for all patients enrolled in the study.

Ethics and dissemination Ethics approval for this study was granted by the Institutional Review Board of Asan Medical Center (no. 2017-0060). Informed consent will be obtained from every participant. The study findings will be published in peer-reviewed journal articles and disseminated through public forums and academic conference presentations. Cost-effectiveness and secondary imaging analyses will be shared in secondary papers.

Trial registration number NCT02978456.
Strengths and limitations of this study

- For the first time, the Quantitative Coronary Angiography versus Intravascular Ultrasound Guidance for Drug-Eluting Stent Implantation trial will evaluate the potential of standardised quantitative coronary angiography (QCA)-based percutaneous coronary intervention (PCl) algorithm into clinical context.

- A practical protocol of QCA-guided PCI has been developed for the trial.

- The trial uses a pragmatic design with inclusion criteria designed to capture a broad range of realworld patients with diverse clinical and anatomical features.

- Bias in event ascertainment may not be ruled out given the open-label trial design.

\section{INTRODUCTION}

Intravascular ultrasound (IVUS) is a useful tool for assessing preintervention lesion characteristics and optimising stent implantation. ${ }^{1}$ Randomised trials evaluating the utility of IVUS for guiding percutaneous coronary intervention (PCI) with drug-eluting stents (DES) over the conventional angiographybased PCI reported conflicting results. Some studies showed better outcomes in patients undergoing IVUS-guided PCI than in those undergoing angiography-guided $\mathrm{PCI},{ }^{2-7}$ while others showed comparable outcomes between the two strategies. ${ }^{8-10}$ In a metaanalysis of these trials, IVUS-guided PCI, by using established criteria for optimising stent deployment, was associated with a reduction in major adverse cardiac events. ${ }^{11-14}$ 
However, in these trials, angiography guidance was based on visual estimation, and high-pressure postdilation with a non-compliant balloon was not routinely used after DES implantation. The visual assessment of coronary artery lesions has a high degree of variability, leading to improper stent sizing with suboptimal stent expansion. ${ }^{15}$ Although the benefit of IVUS-guided PCI is increasingly recognised, its adoption remains low worldwide. ${ }^{16}$ The real barrier to implementing an IVUS programme in daily PGI practice is its high cost. ${ }^{17}$ IVUS is not affordable for many catheterisation laboratories and patients, particularly in low-income and middle-income countries. Thus, a more practical and standardised algorithmic approach to supporting coronary measurement is necessary. On-line quantitative coronary angiography (QCA) is available at every catheterisation laboratory and enables a reliable assessment of lumen diameter without any additional cost. ${ }^{18} 19$ Coronary sizing by on-site QCA may overcome the limitations of visual estimation and aid in deploying the proper DES size.

It is well established that postprocedural minimal lumen diameter determined by angiography, which correlates with the final minimal stent area (MSA) on IVUS, is the key determinant of DES failure. ${ }^{2021}$ Undersizing lumen diameter by visual estimation often leads to the selection of a smaller DES, and the lack of highpressure postdilatation with a non-compliant balloon is frequently related to postprocedural residual stenosis. ${ }^{22}$ DES failure is attributable not to the angiography guidance itself but rather to the suboptimal results associated with underestimated stent sizing by visual estimation and lack of adequate high-pressure postdilatation. We hypothesised that choosing the appropriate DES size by a novel on-site QCA-based algorithm and routine incorporation of high-pressure postdilation with an adequately sized non-compliant balloon may attenuate the disadvantage of the traditional angiography-guided PCI.

\section{METHODS AND ANALYSIS \\ Study overview and objectives}

The Quantitative Coronary Angiography versus Intravascular Ultrasound GUIDancE for Drug-Eluting Stent Implantation (GUIDE-DES) trial is a prospective, multicentre, open-labelled, randomised comparison trial. This trial is investigator initiated with grant support from Biotronik (Bülach, Switzerland). Otherwise, the company will not be involved in any aspect of the study process, including the protocol development, site selection, data collection or data analysis. This study is based on the principles outlined in the Declaration of Helsinki. The primary aim of the trial is to determine whether an on-site QCAbased strategy for PCI guidance is valid for preventing device-oriented events compared with the IVUS-based PCI strategy in all-comer patients who require revascularisation therapy for significant coronary artery disease. The primary analysis will be a non-inferiority comparison of the two strategies for the primary end point of targetlesion failure. The study design is shown in figure 1.

\section{Study population and randomisation}

All consecutive patients with significant native coronary artery disease suitable for DES implantation will be screened for study entry. Patients meeting all the eligibility criteria and providing written informed consent will be included in the study. We will not impose restrictions regarding the clinical diagnosis (chronic or acute coronary syndrome) or location, length, or numbers of lesions to validate the QCA-based PCI algorithm in various PCIindicated patients. However, we will exclude bypass graft

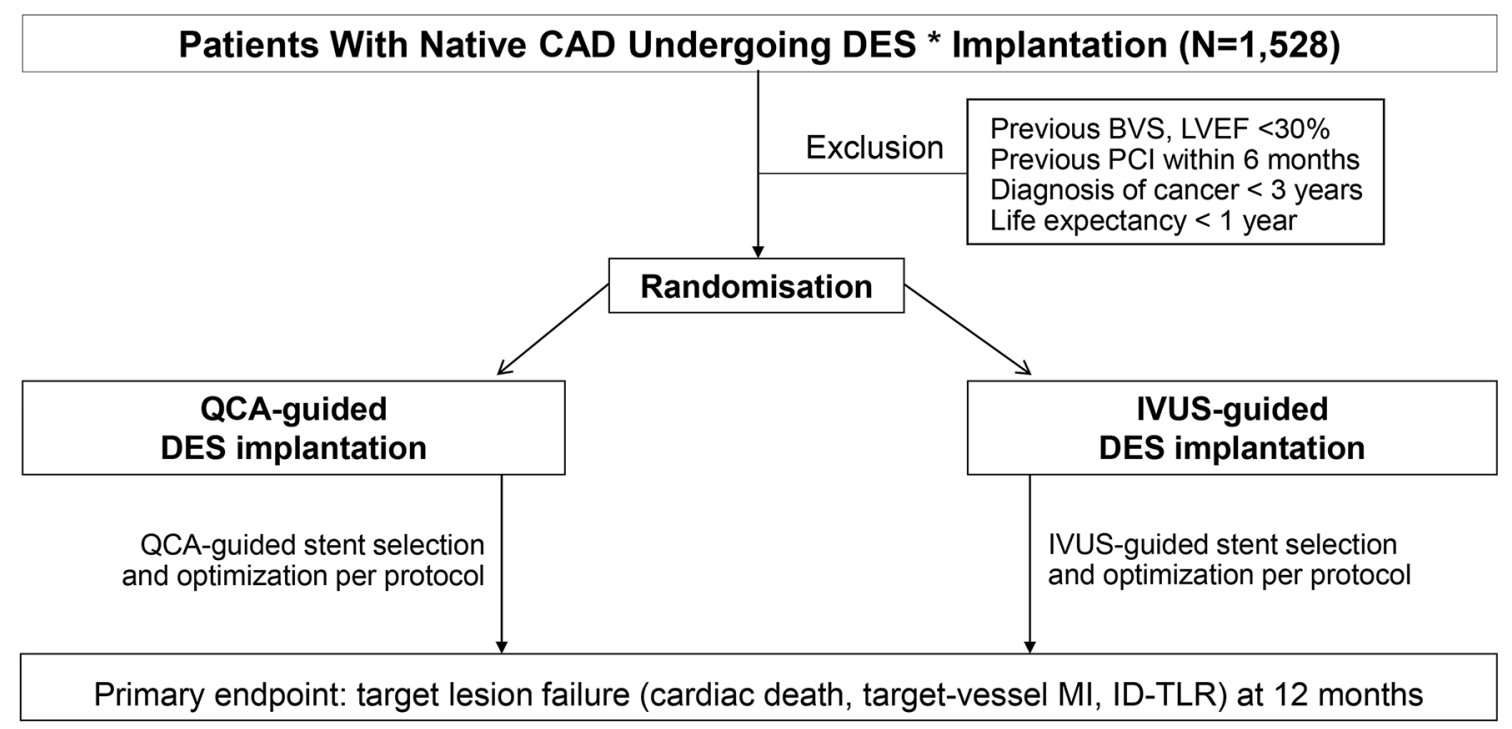

Figure 1 Study flow chart. *Sirolimus-eluting Orsiro or Orsiro mission stents were used in this trial. BVS, bioresorbable vascular scaffold; CAD, coronary artery disease; DES, drug-eluting stent; ID-TLR, ischaemia-driven target-lesion revascularisation; IVUS, intravascular ultrasound; LVEF, left ventricular ejection fraction; MI, myocardial infarction; PCI, percutaneous coronary intervention; QCA, quantitative coronary angiography. 
lesions, for which QCA is less established, and lesions where IVUS delivery is deemed to be impaired (extreme angulation or tortuosity, heavy calcification proximal to or within the target lesion). Detailed information on the inclusion and exclusion criteria is provided in table 1 .

Each patient will receive oral and written information and be required to provide written informed consent at the time of enrolment. Patients will be randomised in a 1:1 ratio to undergo a QCA-guided strategy or an IVUSguided strategy immediately after the guidewire crosses the culprit lesion. The allocation of the study participants will proceed through an Interactive Web Response System with a permutated block size of six. A total of 1528 patients will be enrolled from six high-volume PCI centres in Korea.

\section{Study procedure}

\section{QCA-guided strategy}

A PCI protocol for the QCA-guided group is summarised in figure 2 , and a representative case is illustrated in online supplemental figure 1. An algorithm for the reference segments' diameter adjustment in this trial was developed based on the previous reports comparing lumen measurements between QCA and IVUS. ${ }^{182}$ Applying this, angiograms of vessels that are adequately filled with contrast should be obtained after administering intracoronary nitroglycerin $(250-500 \mu \mathrm{g})$. The best image that corresponds to the lesion location with the least foreshortening should be selected. Lumen diameters are measured at the optimal proximal and distal reference segments by on-site QCA using the automatic calibration software embedded in the angiography systems. If multiple measurements of QCA are performed in different views, it is recommended that the largest value be used for the target diameter calculation. The following formula derives the adjusted QCA value (target diameter) to guide stent selection and deployment: Adjusted QCA value=measured QCA value $+5 \%-10 \%$ of the measured QCA value. Specifically, the percentage number multiplied for the adjustment varies according to the measured QCA value: $10 \%$ for QCA values $\leq 3.5 \mathrm{~mm}, 9 \%$ for $3.6 \mathrm{~mm}, 8 \%$ for $3.7 \mathrm{~mm}$, $7 \%$ for $3.8 \mathrm{~mm}, 6 \%$ for $3.9 \mathrm{~mm}$ and $5 \%$ for QCA values $\geq 4.0 \mathrm{~mm}$. A simple calculation table that can practically be used in the catheterisation lab will be provided to the participating centres (online supplemental table 1). For diffuse disease without a normal-looking segment for the QCA measurement at a bifurcation site, the use of Finet's formula is recommended to estimate the reference lumen diameter of the main branch if applicable (online supplemental figure 2). ${ }^{24}$

The stent size is then selected based on the calculated target diameter of the distal reference segment. The stent length is visually estimated with the aid of a radiopaque guidewire tip $(30 \mathrm{~mm})$ for long lesions or an uninflated balloon (15 or $20 \mathrm{~mm}$ ) for short lesions. During stent deployment, the stent balloon should be inflated up to the pressure corresponding to the distal reference segment's target diameter. Poststenting optimisation
Table 1 Inclusion and exclusion criteria

\section{Inclusion criteria}

\begin{tabular}{|c|c|}
\hline 1. & Man or woman at least 18 years of age \\
\hline 2 & $\begin{array}{l}\text { Typical chest pain or objective evidence of } \\
\text { myocardial ischaemia }\end{array}$ \\
\hline 3. & $\begin{array}{l}\text { Significant stenotic lesions in native coronary } \\
\text { arteries }^{\star} \text { suitable for DES implantation }\end{array}$ \\
\hline 4 & $\begin{array}{l}\text { The patient or guardian agrees to the study } \\
\text { protocol and the schedule of clinical follow-up and } \\
\text { provides written informed consent as approved by } \\
\text { the appropriate institutional review board/ethical } \\
\text { committee of the respective clinical site. }\end{array}$ \\
\hline
\end{tabular}

\section{Exclusion criteria}

1 Angiographic exclusion criteria:

1. Bypass graft lesions

2. Lesions in which impaired delivery of IVUS is expected:

- Extreme angulation $\left(\geq 90^{\circ}\right)$ proximal to or within the target lesion.

- Excessive tortuosity ( $2 \geq 45^{\circ}$ angles) proximal to or within the target lesion.

- Heavy calcification proximal to or within the target lesion.

2 Previous $\mathrm{PCl}$ within 6 months before the index procedure.

3 Previous bioresorbable vascular scaffold implantation.

$4 \quad$ Left ventricular ejection fraction $<30 \%$.

5 Hypersensitivity or contraindication to the device material and its degradants and cobalt, chromium, nickel, platinum, tungsten, acrylic and fluoro polymers that cannot be adequately premedicated.

6 Persistent thrombocytopenia (platelet count $<100$ $\times 10^{9} / \mathrm{L}$

7 Any history of haemorrhagic stroke or intracranial haemorrhage, transient ischaemic attack or ischaemic stroke within the past 6 months.

8 A known intolerance to antiplatelet agents (aspirin, clopidogrel, prasugrel or ticagrelor).

9 Any surgery requiring discontinuation of aspirin and/or use of a P2Y12 inhibitor planned within 12 months after the procedure.

10 A diagnosis of cancer (other than superficial squamous or basal cell skin cancer) in the past 3 years or current treatment for the active cancer.

11 Any clinically significant abnormality identified at the screening visit, physical examination, laboratory tests or ECG which, in the judgement of the investigator, would preclude safe completion of the study.

12 A hepatic disease or biliary tract obstruction, or significant hepatic enzyme elevation (alanine transaminase or aspartate transaminase $>3$ times the upper limit of normal).

13 Life expectancy $<1$ year for any non-cardiac or cardiac causes. 


\section{Table 1 Continued}

\section{Inclusion criteria}

14 Unwillingness or inability to comply with the procedures described in this protocol.

15 Pregnant, breast feeding or childbearing potential.

${ }^{*}$ At least $70 \%$ diameter stenosis on visual estimation, or $50 \%-69 \%$ diameter stenosis with objective evidence of ischaemia (positive non-invasive stress test or fractional flow reserve $\leq 0.8$ ). .DES, drug-eluting stent; IVUS, intravascular ultrasound; PCI, percutaneous coronary intervention

is mandatory using a non-compliant balloon of proper size considering the target diameter of the proximal and distal reference segment. Proximal and distal edge optimisation is performed in which the radiopaque marker of a non-compliant balloon is positioned over the stent edge and the balloon dilated up to the target diameters (online supplemental figure 3). Multiple balloon dilations within the stent should be performed until adequate stent expansion is achieved, preferably assessed by stent boost subtract imaging. If the result is considered suboptimal, the use of a step-up approach with upsizing postdilations (previous ballooning size +about $0.2 \mathrm{~mm}$ ) is recommended. In patients receiving additional stent implantation to treat a dissection at the distal stent edge, postdilation of the overlapping zone with a balloon adequately sized to the proximal stent is needed to eliminate inter-stent malapposition at the overlapping site. This QCA-based PCI algorithm is applicable to main epicardial arteries and side branches and can also be used for the 2-stent technique. The ideal final result would be a harmonious appearance between the reference segment and the stent without dissection and minimal residual stenosis $(<10 \%)$ on angiography. ${ }^{25}$

\section{IVUS-guided strategy}

IVUS can be iteratively used at any step of PCI. After the intracoronary injection of nitroglycerin, the $40-\mathrm{MHz}$ IVUS catheter (Boston Scientific Corporation, Natick, Massachusetts, USA) is advanced more than $5 \mathrm{~mm}$ distal

\begin{tabular}{l}
\hline Design by angiogram \\
- obtain the best angiographic images adequately filled with contrast media \\
- identify the landing zones (normal or normal-looking area) \\
Sizing by QCA \\
- measure the lumen diameter at the reference segments by QCA \\
- calculate the adjusted QCA diameter (target diameter) \\
= measured QCA value $+5 \sim 10 \%$ of the measured QCA value \\
Finish by post-dilation \\
- Stent selection \& deployment: choose the stent size to reach the target diameter of \\
the distal reference segment and inflate the stent balloon up to the target diameter \\
- Stent optimization at its edge and within the stent: high-pressure post-dilation to \\
achieve minimal residual stenosis (diameter stenosis $<10 \%$ ) assessed by stent boost \\
imaging
\end{tabular}

Figure 2 Outline of the QCA-guided PCI strategy. PCI, percutaneous coronary intervention; QCA, quantitative coronary angiography. to the target lesion and withdrawn at a motorised pullback speed of $0.5 \mathrm{~mm} / \mathrm{s}$. Balloon dilatation at the target lesion is allowed to facilitate the IVUS catheter passage if necessary. Stent size and length are determined by the online IVUS measurements. The stent size nearest the distal reference segment's lumen diameter is selected, and the stent length is decided by measuring the distance from the distal to proximal reference sites. During stent deployment, the stent balloon should be inflated up to the pressure corresponding to the mid-wall (or lumen) diameter of the distal reference segment. Adjunctive highpressure balloon dilation using a noncompliant balloon is left to the operator's discretion based on the IVUS findings. It is mandatory to perform IVUS after PCI to assess stent optimisation. The IVUS criteria for stent optimisation in this trial are as follows: (1) in-stent minimal lumen cross-sectional area >distal reference segment's lumen cross-sectional area; (2) complete stent apposition and (3) no significant proximal or distal edge dissection (media dissection, dissection angle $\geq 60^{\circ}$, or dissection length $>2 \mathrm{~mm}$ ). ${ }^{326}{ }^{27}$ If the IVUS-defined optimal criteria are not met, additional procedures are needed.

\section{Study stent and medical treatment}

Biodegradable polymer sirolimus-eluting stents (Orsiro or Orsiro Mission, Bülach, Switzerland, Biotronik) will be used in both trial arms. Optimal angioplasty requires compliance with precise guidelines for adjunctive pharmacological therapy. Unless pretreated, all patients should be administered aspirin $300 \mathrm{mg}$ and P2Y12 inhibitors (clopidogrel $600 \mathrm{mg}$, ticagrelor $180 \mathrm{mg}$, prasugrel 60 $\mathrm{mg}$ ) before PCI. Unfractionated heparin must be administered before and during the procedure to maintain an activated clotting time greater than $250 \mathrm{~s}$. According to the clinical indication and procedural complexity, dual antiplatelet agents will be prescribed for at least 6-12 months following PCI at the discretion of the attending physician, and either aspirin (100 mg once daily) or clopidogrel (75 mg once daily) will be continued indefinitely thereafter.

Other pharmacological treatments must be optimised early after randomisation in accordance with the established standards of practice. ${ }^{28} 29$ Statins should be prescribed in all patients during the study period. Betablockers, calcium channel blockers or long-acting nitrates alone or in combination can be used as antiischaemic therapy. An ACE inhibitor or an angiotensin-receptor blocker is considered for secondary prevention. Blood pressure and diabetic control are emphasised. Patients should receive counselling about smoking cessation, weight control and regular exercise.

\section{Study endpoints and follow-up}

The primary endpoint is the 12-month cumulative incidence of target-lesion failure defined as a composite of cardiac death, target-vessel myocardial infarction or ischaemia-driven target-lesion revascularisation. Secondary endpoints are the rates of all-cause death, 


\section{Box 1 Primary and secondary endpoints}

\section{Primary endpoint}

- Target-lesion failure (composite of cardiac death, target vessel myocardial infarction or ischaemia-driven target lesion revascularisation) at 12 months after randomisation

\section{Secondary endpoints \\ - Procedural success \\ - Death at 12 months \\ - Myocardial infarction at 12 months \\ - Stent thrombosis (definite/probable) at 12 months \\ - Stroke at 12 months \\ - Target-lesion revascularisation at 12 months \\ - Any revascularisation at 12 months \\ Economic (cost-effectiveness) analysis at 12 months}

myocardial infarction, definite or probable stent thrombosis, stroke, target-lesion revascularisation and any revascularisation at 12 months and procedural success (box 1). A cost-effectiveness comparison of QCA-guided versus IVUS-guided DES implantation will be performed independently. Procedural success is defined as the achievement of final in-stent residual stenosis of less than $30 \%$ by QCA of at least one stent at the intended target lesion and successful withdrawal of the delivery system for all target lesions without the occurrence of cardiac death, target-vessel myocardial infarction or repeat target-lesion revascularisation during the hospital stay. All deaths will be considered cardiac unless an unequivocal non-cardiac cause can be established. Specifically, any unexpected death even in patients with coexisting potentially fatal non-cardiac disease (eg, cancer, infection) is classified as cardiac. The diagnosis of periprocedural myocardial infarction is based on the diagnostic criteria from the Society for Cardiovascular Angiography and Interventions. ${ }^{30}$ The diagnosis of spontaneous myocardial infarction is based on criteria proposed by the Third Universal Definition of Myocardial Infarction. ${ }^{31}$ Stroke is defined as focal loss of neurological function caused by an ischaemic or haemorrhagic event, with residual symptoms lasting at least 24 hours or leading to death. Target-lesion revascularisation is defined as any repeat PCI of the target lesion or bypass surgery of the target vessel performed for restenosis or other complication of the target lesion.

Clinical follow-up assessments will be scheduled via clinical visits or telephone interviews at 1, 6 and 12 months for all patients enrolled in the study. Medical history will be obtained, while a physical examination and basic laboratory tests will be performed at each visit. Data collected during the follow-up visits will include ischaemic symptoms, bleeding complications and major adverse cardiac events, including rehospitalisation and recatheterisation. Angiographic and IVUS images will be collected at the core laboratory of Asan Medical Center and analysed offline by experts blinded to clinical data.

\section{Statistical analysis}

This trial will test the hypothesis that QCA-guided PCI is non-inferior to IVUS-guided PCI concerning the primary end point of cardiac death, target-vessel myocardial infarction or ischaemia-driven target vessel revascularisation at 12 months. Based on previous reports of realworld patients without restrictions regarding the clinical diagnosis; lesion number, severity or location; or number of stents used, ${ }^{32} 33$ we estimated that the incidence of the primary endpoint 12 months after the index procedure would be $8 \%$ in the IVUS-guided PCI group. Using a noninferiority margin of $3.5 \%$ in accordance with the noninferiority margins used in contemporary trials of DES and considering a $5 \%$ of attrition rate, we estimated that with a total of 1528 patients, the study would provide $80 \%$ power to show noninferiority on the basis of the likelihood-score method by Farrington and Manning at a one-sided 0.025 level. $^{34} 35$

The analyses will be performed according to the intention-to-treat principle. A secondary per-protocol analysis will be performed to assess the effect of treatment crossovers or unanticipated problems that could dilute treatment differences of interest. Continuous variables will be presented as mean and SD, while categorical variables will be shown as numbers and percentages. Intergroup differences will be evaluated by Student's t-test or the Wilcoxon rank sum test for continuous variables and by Pearsons's $\chi^{2}$ test or Fisher's exact test for categorical variables as appropriate. Cumulative event rates and survival curves will be generated using the Kaplan-Meier method, while intergroup differences will be compared by the log-rank test. Follow-up will be censored at the date of the last follow-up or at 1 year, whichever comes first. Cox's proportional hazards regression analyses will be conducted to estimate the risk associated with the QCAguided PCI strategy relative to that with the IVUS-guided PCI strategy. The proportional hazards assumption about the assigned treatments will be tested with the Schoenfeld residuals test. A two-sided $\mathrm{p}<0.05$ will indicate significance. SAS software V.9.3 (SAS Institute) will be used for all the statistical analyses.

\section{Trial organisation}

The members of the executive committee include the principal investigators of the investigating centres and the persons who organised this study. The committee approved the final trial design and protocol issued to the data and safety monitoring board (DSMB) and the clinical sites. The committee will be responsible for reviewing the final results, determining the methods of presentation and publication, and selecting secondary projects and publications by members of the steering committee. An independent DSMB committee, headed by Sung Cheol Yun, will receive information on rates of death, myocardial infarction, and major bleeding and will make recommendations based on the analyses of safety data, protocol deviation, IVUS failures and 30-day follow-up reports. The DSMB chairperson will notify the data 
coordinating centre of any safety or compliance issues. The committee will also provide confidential recommendations as necessary of study termination based on the safety stopping rules determined at the study onset or when a clinically significant result is identified in safety analyses of the data. This study will not be stopped early based on efficacy results. The executive committee has right to the final decision to stop the study prematurely based on DSMB recommendations. All DSMB reports will remain strictly confidential, but will be made available to the regulatory body on request. The centralised clinical events committee (CEC) is made up of interventional and non-interventional cardiologists who are not participants in the study. The CEC develops specific criteria used to categorise clinical events in the study that are based on the protocol. At the trial onset, the CEC will establish clear rules outlining the minimum amount of data required and the algorithm followed to classify a clinical event. All members of the CEC will be blinded to the primary results of the trial. Data coordination and site management services will be performed by the Clinical Research Center of Asan Medical Center, Seoul, Korea.

\section{Patient and public involvement}

There was no patient or public involvement in this trial.

\section{Ethical approval and dissemination}

The study protocol was approved by the internal review board of Asan Medical Center, Seoul, Korea (no. 20170060) and each participating centre. The current protocol version is 3.2, date 11 March 2021. Informed consent will be obtained from every participant by study personnel. The authors are solely responsible for this study's design and conduct, all study analyses, manuscript drafting and editing, and final manuscript contents. The study findings will be published in peer-reviewed journal articles and disseminated through public forums and academic conference presentations. Cost-effectiveness and secondary imaging analyses will be shared in secondary papers.

\section{Current status}

The anticipated duration of the study is approximately 48 months, including the enrolment period of 30 months and the follow-up period of 12 months. The first patient was enrolled on 23 February 2017, and 1338 patients were recruited at the end of March 2021. Although patient inclusion has been delayed due to the ongoing COVID-19 outbreak, enrolment is expected to end by September 2021. The primary result of the GUIDE-DES trial will be available by late 2022 or early 2023 .

\section{DISCUSSION}

With IVUS guidance, acute stent placement can be optimised toward more significant stent expansion and fewer stent edge problems based on the reliable information about vessel size, plaque burden, suboptimal stent deployment and procedure-related complications. To date, 10 randomised trials have compared IVUS-guided DES implantation with conventional angiographical guidance. In the IVUS group of one trial, the achievement of a minimum stent cross-sectional area greater than the distal reference lumen with IVUS guidance was associated with a $2.9 \%$ rate of 1-year major adverse cardiac events vs $5.8 \%(\mathrm{p}=0.007)$ with angiography guidance. ${ }^{3}$ Another large-scale trial showed that by achieving an MSA $>5.0 \mathrm{~mm}^{2}$ and avoiding geographic miss, IVUS guidance significantly reduced the rate of target-vessel failure at 1 year. ${ }^{7}$ However, despite the accumulating evidence supporting the use of IVUS to improve outcomes after PCI, its use continues to be infrequent worldwide, mostly because of the inaccessibility related to high device cost or image interpretation inexperience. ${ }^{36}$ Thus, an overlooked unmet need regarding PCI is to find a way to improve outcomes of DES in a typical circumstance when IVUS is not available. An important step forward would be developing a method to overcome the drawback of conventional angiography-guided PCI. Our study has incorporated QCA into clinical context and developed a novel size selection algorithm based on the QCA measurement, which standardises the angiography-based PCI procedure to select an appropriately sized stent or balloon without significant intraindividual or interindividual variability.

Previous randomised trials did not provide an objective guide or definition for stent optimisation for the angiography-guided group. Using visual assessment, interventionists tend to choose undersized stents and perform less aggressive postdilation, leading to suboptimal immediate results and an increased risk of target-lesion failure. ${ }^{37}$ QCA has been used to provide quantitative measures of angiography, mostly in clinical studies. The advantage of QCA over visual estimation is that its measurements are objective and relatively reproducible. Furthermore, QCA is easy to use without coregistration or additional cost and is available at every catheterisation laboratory. Unfortunately, it is not commonly used to guide PCI in real-world practice. This trial will test the utility of real-time QCA guidance for PCI with a goal of incorporating core laboratory experiences into daily clinical practice.

In the PROSPECT substudy, there was a strong correlation between minimal lumen diameters on QCA and IVUS, with underestimation in relatively small arteries $(<3.8 \mathrm{~mm})$ and overestimation in larger arteries $(>3.8$ $\mathrm{mm})$ with an excellent correlation $(\mathrm{r}=0.89, \mathrm{p}<0.001){ }^{23}$ Optical coherence tomography (OCT) accurately measures lumen diameters because it produces highresolution images that are identical to the actual values. The OPUS-CLASS study showed that QCA underestimates lumen diameters by $5 \%$ compared with OCT, whereas IVUS overestimates lumen diameters by $8 \%$ compared with OCT. ${ }^{18}$ Therefore, in this study, we planned to differentially adjust the measured QCA values by $5 \%-10 \%$ to estimate the reference segment's lumen diameter. Inadequate filling of the vessels with contrast 
media and coronary artery spasms lead to underestimation of the accurate lumen dimensions. Thus, taking images of vessels filled with contrast medium after nitroglycerin injection is recommended to overcome measurement errors. QCA should be repeated if the coronary lumen dimension increases after pre-dilation of severely stenotic lesions. The American College of Cardiology/ American Heart Association guideline recommends a minimum residual percent diameter stenosis of $<10 \%$ by visual estimation after stent implantation, and this criterion as stent optimisation was adopted in the QCA-guided arm in our study. The concept of 'the bigger, the better' remains valid in the DES era. Contemporary thin-strut DES may have weaker radial strength and greater recoil with a smaller lumen area, requiring the need for highpressure postdilation to achieve optimal PCI results. ${ }^{37}$ Stent boost subtract imaging allows clear visualisation of the stents and reliable detection of stent underexpansion. ${ }^{38}$ Routine high-pressure postdilation, preferably guided by stent boost subtract imaging, will likely lead to minimal residual diameter stenosis with a low risk of edge problems. ${ }^{22}{ }^{39}$ The GUIDE-DES trial will explore whether incorporating these angiography-based technical considerations into a standardised PCI algorithm may be an acceptable alternative to IVUS-guided PCI in terms of device-oriented PCI outcomes.

The success of using QCA for real-time PCI guidance may have significant future implications along with the development of artificial intelligence technologies. A robust deep learning model has already been proposed to automatically segment the major vessels on coronary angiography. ${ }^{40}$ With this technique, the image processing time can be minimised with less manual correction, allowing immediate QCA analysis on the operator screen in the catheterisation room. Thus, diagnosis with 3-D QCA could be utilised for PCI by combining the 2-D QCA of multiple angiograms. ${ }^{41}$ Further investigations of IVUSbased machine learning algorithms may lead to outcomes similar to those with IVUS guidance after QCA-guided PCI.

\section{Author affiliations}

${ }^{1}$ Division of Cardiology, Department of Internal Medicine, Asan Medical Center, University of Ulsan College of Medicine, Songpa-gu, Seoul, Korea (the Republic of) ${ }^{2}$ Department of Cardiology, Cardiovascular Center, Korea University Anam Hospital, Seoul, Korea (the Republic of)

${ }^{3}$ Department of Cardiology, Hallym University Sacred Heart Hospital, Anyang, Korea (the Republic of)

${ }^{4}$ Division of Cardiology, Gangnam Severance Hospital, Yonsei University College of Medicine, Seoul, Korea (the Republic of)

${ }^{5}$ Division of Cardiology, Department of Internal Medicine, Kangbuk Samsung Hospital, Sungkyunkwan University School of Medicine, Seoul, Korea (the Republic of)

${ }^{6}$ Department of Cardiology, National Health Insurance Service Ilsan Hospital, Gyeonggi-do, Korea

Acknowledgements We thank all members of this trial group for their ideas, suggestions, participation and general assistance. This paper was edited for language by Editage (www.editage.co.kr).

Contributors PHL, S-WL and CWL developed the trial concept and wrote the protocol and the manuscript of the protocol publication. SJH, H-SK, YWY, J-YL,
S-JO, S-JK, Y-HK and S-WP helped to develop the trial concept and revised the manuscript critically for important intellectual content.

Funding This study is funded by an unrestricted grant from Biotronik, Bülach, Switzerland (G1709).

Competing interests None declared.

Patient consent for publication Not applicable.

Provenance and peer review Not commissioned; externally peer reviewed.

Supplemental material This content has been supplied by the author(s). It has not been vetted by BMJ Publishing Group Limited (BMJ) and may not have been peer-reviewed. Any opinions or recommendations discussed are solely those of the author(s) and are not endorsed by BMJ. BMJ disclaims all liability and responsibility arising from any reliance placed on the content. Where the content includes any translated material, BMJ does not warrant the accuracy and reliability of the translations (including but not limited to local regulations, clinical guidelines, terminology, drug names and drug dosages), and is not responsible for any error and/or omissions arising from translation and adaptation or otherwise.

Open access This is an open access article distributed in accordance with the Creative Commons Attribution Non Commercial (CC BY-NC 4.0) license, which permits others to distribute, remix, adapt, build upon this work non-commercially, and license their derivative works on different terms, provided the original work is properly cited, appropriate credit is given, any changes made indicated, and the use is non-commercial. See: http://creativecommons.org/licenses/by-nc/4.0/.

ORCID iDs

Soon Jun Hong http://orcid.org/0000-0003-4832-6678

Cheol Whan Lee http://orcid.org/0000-0003-3987-2159

\section{REFERENCES}

1 Hibi K, Kimura K, Umemura S. Clinical utility and significance of intravascular ultrasound and optical coherence tomography in guiding percutaneous coronary interventions. Circ J 2015;79:24-33.

2 Chieffo A, Latib A, Caussin C, et al. A prospective, randomized trial of intravascular-ultrasound guided compared to angiography guided stent implantation in complex coronary lesions: the AVIO trial. Am Heart J 2013;165:65-72.

3 Hong S-J, Kim B-K, Shin D-H, et al. Effect of intravascular ultrasound-guided vs angiography-guided everolimus-eluting stent implantation: the IVUS-XPL randomized clinical trial. JAMA 2015;314:2155-63.

4 Tian N-L, Gami S-K, Ye F, et al. Angiographic and clinical comparisons of intravascular ultrasound- versus angiographyguided drug-eluting stent implantation for patients with chronic total occlusion lesions: two-year results from a randomised AIR-CTO study. Eurolntervention 2015;10:1409-17.

5 Tan Q, Wang Q, Liu D, et al. Intravascular ultrasound-guided unprotected left main coronary artery stenting in the elderly. Saudi Med J 2015;36:549-53.

6 Mariani J, Guedes C, Soares P, et al. Intravascular ultrasound guidance to minimize the use of iodine cOntrast in percutaneous coRonary intervention: the MOZART (minimizing cOntrast utiliZation with IVUS guidance in coRonary angioplasTy) randomized controlled trial. JACC Cardiovasc Interv 2014;7:1287-93.

7 Zhang J, Gao X, Kan J, et al. Intravascular Ultrasound Versus Angiography-Guided Drug-Eluting Stent Implantation: The ULTIMATE Trial. J Am Coll Cardiol 2018;72:3126-37.

8 Jakabcin J, Spacek R, Bystron M, et al. Long-Term health outcome and mortality evaluation after invasive coronary treatment using drug eluting stents with or without the IVUS guidance. randomized control trial. home des IVUS. Catheter Cardiovasc Interv 2010;75:578-83.

$9 \mathrm{Kim}$ J-S, Kang T-S, Mintz GS, et al. Randomized comparison of clinical outcomes between intravascular ultrasound and angiography-guided drug-eluting stent implantation for long coronary artery stenoses. JACC Cardiovasc Interv 2013;6:369-76.

10 Kim B-K, Shin D-H, Hong M-K, et al. Clinical impact of intravascular ultrasound-guided chronic total occlusion intervention with zotarolimus-eluting versus Biolimus-Eluting stent implantation: randomized study. Circ Cardiovasc Interv 2015;8:e002592.

11 Steinvil A, Zhang Y-J, Lee SY, et al. Intravascular ultrasound-guided drug-eluting stent implantation: an updated meta-analysis of randomized control trials and observational studies. Int J Cardiol 2016;216:133-9.

12 Elgendy IY, Mahmoud AN, Elgendy AY, et al. Outcomes with intravascular ultrasound-guided stent implantation: a meta-analysis 
of randomized trials in the era of drug-eluting stents. Circ Cardiovasc Interv 2016:9:e003700.

13 Shin D-H, Hong S-J, Mintz GS, et al. Effects of Intravascular Ultrasound-Guided Versus Angiography-Guided New-Generation Drug-Eluting Stent Implantation: Meta-Analysis With Individual Patient-Level Data From 2,345 Randomized Patients. JACC Cardiovasc Interv 2016;9:2232-9.

14 Bavishi C, Sardar P, Chatterjee S, et al. Intravascular ultrasoundguided vs angiography-guided drug-eluting stent implantation in complex coronary lesions: meta-analysis of randomized trials. Am Heart J 2017;185:26-34.

15 Campbell PT, Mahmud E, Marshall JJ. Interoperator and intraoperator (in)accuracy of stent selection based on visual estimation. Catheter Cardiovasc Interv 2015;86:1177-83.

16 Smilowitz NR, Mohananey D, Razzouk L, et al. Impact and trends of intravascular imaging in diagnostic coronary angiography and percutaneous coronary intervention in inpatients in the United States. Catheter Cardiovasc Interv 2018;92:E410-5.

17 Mintz GS, Guagliumi G. Intravascular imaging in coronary artery disease. Lancet 2017;390:793-809.

18 Kubo T, Akasaka T, Shite J, et al. Oct compared with IVUS in a coronary lesion assessment: the OPUS-CLASS study. JACC Cardiovasc Imaging 2013;6:1095-104.

19 Sotomi Y, Onuma Y, Suwannasom P, et al. Is quantitative coronary angiography reliable in assessing the lumen gain after treatment with the everolimus-eluting bioresorbable polylactide scaffold? Eurolntervention 2016;12:e998-1008.

20 Mercado N, Boersma E, Wijns W, et al. Clinical and quantitative coronary angiographic predictors of coronary restenosis: a comparative analysis from the balloon-to-stent era. J Am Coll Cardiol 2001:38:645-52.

21 Cassese S, Byrne RA, Tada T, et al. Incidence and predictors of restenosis after coronary stenting in 10004 patients with surveillance angiography. Heart 2014;100:153-9.

22 Romagnoli E, Sangiorgi GM, Cosgrave J, et al. Drug-eluting stenting: the case for post-dilation. JACC Cardiovasc Interv 2008;1:22-31.

23 Goto K, Mintz GS, Litherland C, et al. Lumen measurements from quantitative coronary angiography and IVUS: A prospect substudy. JACC Cardiovasc Imaging 2016;9:1011-3.

24 Finet G, Gilard M, Perrenot B, et al. Fractal geometry of arterial coronary bifurcations: a quantitative coronary angiography and intravascular ultrasound analysis. EuroIntervention 2008:3:490-8.

25 Levine GN, Bates ER, Blankenship JC. 2011 ACCF/AHA/SCAI guideline for percutaneous coronary intervention. A report of the American College of cardiology Foundation/American heart association Task force on practice guidelines and the Society for cardiovascular angiography and interventions. J Am Coll Cardiol 2011;124:e44-122.

26 Choi S-Y, Witzenbichler B, Maehara A, et al. Intravascular ultrasound findings of early stent thrombosis after primary percutaneous intervention in acute myocardial infarction: a Harmonizing outcomes with revascularization and stents in acute myocardial infarction (HORIZONS-AMI) substudy. Circ Cardiovasc Interv 2011;4:239-47.

27 Cheneau E, Leborgne L, Mintz GS, et al. Predictors of subacute stent thrombosis: results of a systematic intravascular ultrasound study. Circulation 2003;108:43-7.
28 Amsterdam EA, Wenger NK, Brindis RG, et al. 2014 AHA/ACC guideline for the management of patients with non-ST-elevation acute coronary syndromes: a report of the American College of Cardiology/American heart association Task force on practice guidelines. Circulation 2014;130:e344-426.

29 Fihn SD, Gardin JM, Abrams J, et al. 2012 ACCF/AHA/ACP/AATS/ PCNA/SCAI/STS guideline for the diagnosis and management of patients with stable ischemic heart disease: Executive summary: a report of the American College of cardiology Foundation/American heart association Task force on practice guidelines, and the American College of physicians, American association for thoracic surgery, preventive cardiovascular nurses association, Society for cardiovascular angiography and interventions, and society of thoracic surgeons. Circulation 2012;126:3097-137.

30 Moussa ID, Klein LW, Shah B, et al. Consideration of a new definition of clinically relevant myocardial infarction after coronary revascularization: an expert consensus document from the Society for cardiovascular angiography and interventions (Scal). Catheter Cardiovasc Interv 2014;83:27-36.

31 Thygesen K, Alpert JS, Jaffe AS, et al. Third universal definition of myocardial infarction. Circulation 2012;126:2020-35.

32 Silber S, Windecker S, Vranckx P, et al. Unrestricted randomised use of two new generation drug-eluting coronary stents: 2-year patient-related versus stent-related outcomes from the RESOLUTE all Comers trial. Lancet 2011;377:1241-7.

33 Park D-W, Kim Y-H, Song H-G, et al. Outcomes after unrestricted use of everolimus-eluting and sirolimus-eluting stents in routine clinical practice: a multicenter, prospective cohort study. Circ Cardiovasc Interv 2012;5:365-71.

34 Farrington CP, Manning G. Test statistics and sample size formulae for comparative binomial trials with null hypothesis of non-zero risk difference or non-unity relative risk. Stat Med 1990;9:1447-54.

35 Ellis SG, Kereiakes DJ, Metzger DC, et al. Everolimus-Eluting Bioresorbable scaffolds for coronary artery disease. N Engl J Med 2015;373:1905-15

36 Elgendy IY, Ha LD, Elbadawi A, et al. Temporal Trends in Inpatient Use of Intravascular Imaging Among Patients Undergoing Percutaneous Coronary Intervention in the United States. JACC Cardiovasc Interv 2018;11:913-5.

37 de Ribamar Costa J, Mintz GS, Carlier SG, et al. Intravascular ultrasound assessment of drug-eluting stent expansion. Am Heart $J$ 2007:153:297-303.

38 Mishell JM, Vakharia KT, Ports TA, et al. Determination of adequate coronary stent expansion using StentBoost, a novel fluoroscopic image processing technique. Catheter Cardiovasc Interv 2007;69:84-93

39 Brodie BR, Cooper C, Jones $\mathrm{M}$, et al. Is adjunctive balloon postdilatation necessary after coronary stent deployment? final results from the POSTIT trial. Catheter Cardiovasc Interv 2003;59:184-92.

40 Yang S, Kweon J, Roh J-H, et al. Deep learning segmentation of major vessels in X-ray coronary angiography. Sci Rep 2019;9:16897.

41 Tu S, Xu L, Ligthart J, et al. In vivo comparison of arterial lumen dimensions assessed by co-registered three-dimensional (3D) quantitative coronary angiography, intravascular ultrasound and optical coherence tomography. Int J Cardiovasc Imaging 2012;28:1315-27. 